\title{
Association of COVID-19 with Rhino-Cerebral Mucormycosis: An Observational Study
}

\author{
Varsha Agarwal $^{1} \cdot$ Ambika Gupta $^{1} \cdot$ Virendra Singh $^{2} \cdot$ Neha Jajodia $^{2} \cdot$ \\ Harsha Popli ${ }^{1}$ R. Akilan ${ }^{2}$
}

Received: 30 September 2021 / Accepted: 26 October 2021/Published online: 11 November 2021

(C) The Association of Oral and Maxillofacial Surgeons of India 2021

\begin{abstract}
Mucormycosis is becoming more common in patients infected with or recovering from COVID-19 under the current situation. However, there is a scarcity of research on a specific group of COVID-19 individuals who are predisposed to mucormycosis. As a result, a retrospective observational study was undertaken to assess the possible connection of mucormycosis with COVID-19. The goal was also to investigate the link between diabetes mellitus, mucormycosis and COVID-19. The occurrence and severity of the disease among the case records were assessed using signs and symptoms, investigations conducted during COVID-19, and serological test results. Patients were classified as previously known diabetics, recently diagnosed diabetics, or non-diabetics based on their history of pre-existing DM and HBAlc levels at the time of reporting for mucormycosis. The findings show that mild COVID-19-infected patients and non-diabetic patients have a high incidence of COVID-19-associated mucormycosis (CAM). As a result, all clinicians participating in the care of COVID-infected patients must be aware of the significant risk of secondary mucormycosis in the post-recovery phase, even in those with mild or moderate symptoms.
\end{abstract}

Keywords Mucormycosis · COVID-19 · Invasive fungal infections $\cdot$ Mycoses $\cdot$ Diabetes

Ambika Gupta

drambika79@rediffmail.com

1 Dept. of Oral Medicine and Radiology, Post Graduate Institute of Dental Sciences, Room No. 11, Rohtak 124001, India

2 Dept. Of Oral and Maxillofacial Surgery, Post Graduate Institute of Dental Sciences, Rohtak, India

\section{Introduction}

COVID-19, a disease caused by SARS-CoV-2, was first reported in Wuhan, China, in December 2019 and has spread across the globe since then to become a worldwide pandemic. Respiratory distress is frequently seen with COVID-19. But in a great deal of cases, the virus affects a person's overall health with a myriad of manifestations. Various sequelae and complications are emerging with each passing day [1]. The identification and understanding the pathophysiology behind these spectrum of manifestation are crucial for the overall well-being of the patient.

Mucormycosis or zygomycosis or phycomycosis is a rapid progressing, opportunistic, invasive fungal infection, predominantly seen in immune-compromised individuals with impaired neutrophil response [2]. In the current scenario, this previously rare invasive fungal infection is being seen increasingly, in cases infected with or recovered from COVID-19. A complex interaction of factors, including pre-existing diseases, use of steroids and antibiotics as the management modality for COVID-19-induced complications, the risk of hospital-acquired infections and systemic immune alterations due to COVID-19 infection itself, may have been some of the predisposing factors that led to mucormycosis [3]. But, the exact pathophysiological mechanism underlying this trampling association of COVID-19 and mucormycosis is still not clear. There is paucity of literature regarding the predisposition to mucormycosis in a particular category of COVID-19 patients.

So, the present study was designed with the aim to evaluate the possible association of mucormycosis with COVID-19 infection and it's severity is depicted in Figs. 1, 2. Association of DM and CAM is depicted in Fig. 3 and Table 1 . The objective was also to study the association of 
Fig. 1 Distribution of the diagnosis of COVID-19 infection in the mucormycosis patients

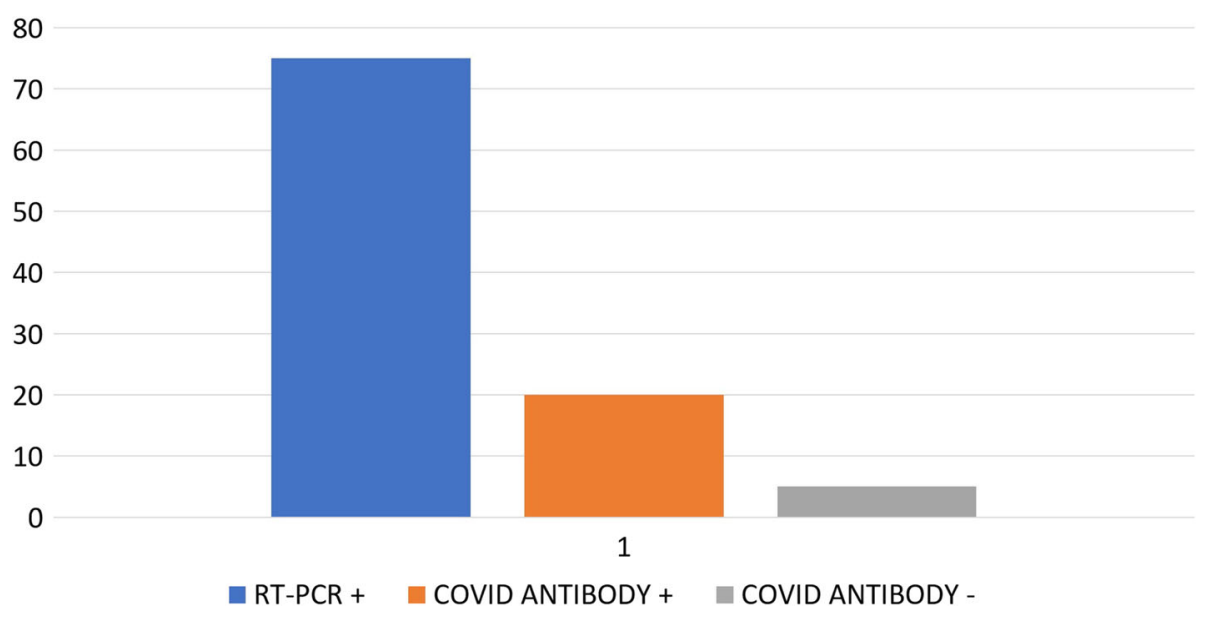

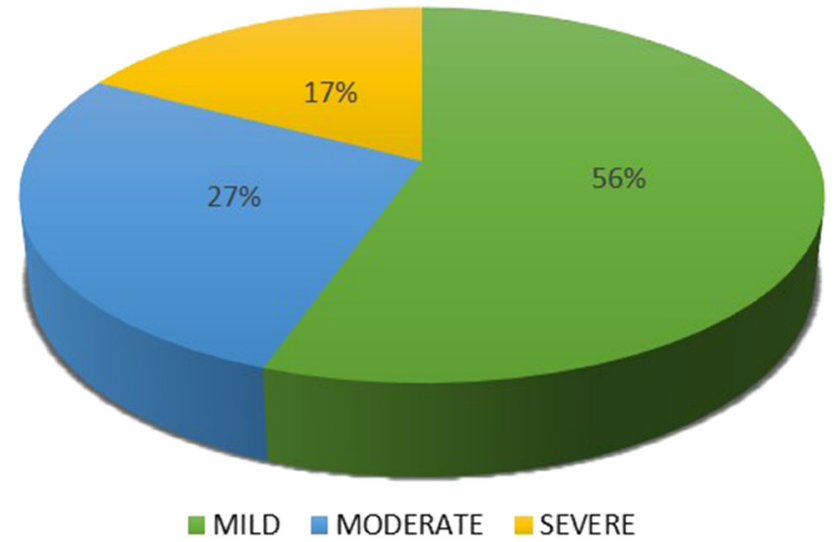

Fig. 2 Categorization of mucormycosis patients according to severity of COVID-19 infection

diabetes mellitus (DM) with COVID-19-associated mucormycosis (CAM).

\section{Materials and Methods}

A retrospective observational study was conducted at a tertiary care dental facility from November 2020 to June 2021. A sample size of 144 was determined, assuming a prevalence of CAM of $90 \%$ and a margin of error of $5 \%$. Records of the patients with rhino-maxillary and rhinoorbito-cerebral mucormycosis who reported to the outpatient and emergency department during this duration were retrieved for the study. The records were analysed for the history of COVID-19 infection. The signs and symptoms and investigations done during the course of COVID-19 were analysed to evaluate the severity of the disease. According to the guidelines issued by the Government of India, the cases were categorised as mild, moderate and severe COVID-19 [4]. In the cases with a negative realtime reverse transcription polymerase chain reaction (RT-
PCR) report for COVID-19, the serological test results were analysed. The patients with a negative history of COVID-19 symptoms were categorised under mild disease, if their serum antibody test was positive, while those with symptoms of COVID-19 were classified as mild, moderate or severe using the same criteria as in RT-PCR-positive cases. Those with a negative serum antibody test were assumed not to be infected with COVID-19. The patients who had received even a single dose of COVID vaccine were excluded from the study so as to avoid any bias. The history of pre-existing DM and HBA1c levels at the time of reporting for mucormycosis was analysed, and the patients were further categorised as previously known diabetics (PD), recently diagnosed diabetics (RD) and non-diabetics (ND). The results were presented as the percentage belonging to each group, based upon their COVID-19 infection severity and diabetic status.

\section{Results}

A total of 147 case records of the patients with mucormycosis were evaluated. $74.8 \%$ patients $(n=110)$ out of 147 gave a history of COVID-19 infection with a positive RT-PCR report. For the remaining $25.2 \%$ case records $(\mathrm{n}=37)$, antibody tests were analysed. $19.73 \%$ patients $(n=29)$ had positive test results, whereas $5.44 \%$ $(\mathrm{n}=8)$ had negative test reports.

Among the $74.8 \%(\mathrm{n}=110)$ RT-PCR positive group, $33.33 \%$ patients $(n=49)$ had a history of mild symptoms, $25.17 \%(\mathrm{n}=37)$ had moderate severity of symptoms and $16.33 \%(\mathrm{n}=24)$ had severe COVID-19 infection.

Among the $19.73 \%(n=29)$ antibody-positive cases, $19.05 \%$ cases $(n=28)$ were asymptomatic and hence was categorised as mild case of COVID-19, and $0.68 \%$ case $(n=1)$ had signs and symptoms of moderate category of infection, but did not test positive for RT-PCR. 
Fig. 3 Spectrum of diabetic status of the patients with mucormycosis

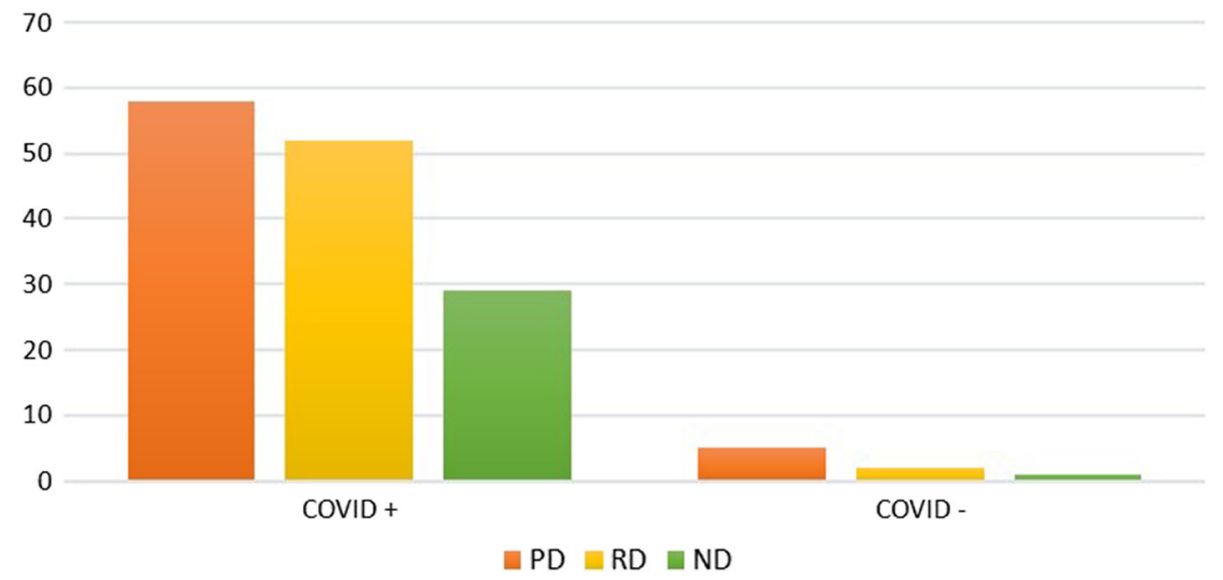

Table 1 Total number of previously diagnosed diabetic, recently diagnosed diabetic and non-diabetic cases among all the categories

Total mucormycosis cases: 147

\begin{tabular}{|c|c|c|c|c|c|c|}
\hline & \multicolumn{3}{|c|}{$\mathrm{RT}-\mathrm{PCR}+: 110$} & \multicolumn{2}{|l|}{ RT-PCR-COVID Antibody $+: 29$} & \multirow{2}{*}{$\begin{array}{l}\text { RT-PCR-COVID Antibody -: } 8 \\
-\end{array}$} \\
\hline & MILD: 49 & MOD: 37 & SEVERE: 24 & MILD (ASYMPTOMATIC): 28 & MOD (SYMPTOMATIC): 1 & \\
\hline PD & 23 & 16 & 10 & 9 & - & 5 \\
\hline $\mathrm{RD}$ & 16 & 15 & 10 & 11 & - & 2 \\
\hline ND & 10 & 6 & 4 & 8 & 1 & 1 \\
\hline
\end{tabular}

*PD: previously known diabetics; RD: recently diagnosed diabetics; ND: non-diabetics

$5.44 \%$ patients $(n=8)$ had both negative RT-PCR and COVID antibody report.

The total number of PD, RD and ND cases among all the categories is displayed in Table 1.

\section{Discussion}

Phycomycosis or zygomycosis was initially described by Paltauf in 1885 and later coined as Mucormycosis by Baker in 1957. It is described as an uncommon and aggressive fungal infection, which usually affects immune-compromised patients. Various risk factors for this invasive fungal infection include diabetes, systemic use of corticosteroid, neutropenia, haematologic malignancies, immunosuppression post-stem cell transplant or organ transplant. It has six types of presentations, which include rhino-cerebral, pulmonary, cutaneous, gastrointestinal, disseminated and miscellaneous [5].

Rhino-cerebral presentation is the most common form. The incidence rate varies from 0.005 to 1.7 per million population in the world. In India, the prevalence rate is about 80 times of that in developed countries, i.e. approx.
0.14 cases per 1000 population. This pre-existing load has seen a rapid surge owing to the COVID-19 pandemic [6].

Presently, the gold-standard method to diagnose SARSCoV-2 is the RT-PCR assay. This test has certain limitations that includes changes in diagnostic accuracy over the disease course and the potential false-negative results [7]. There are a lot of asymptomatic cases of COVID-19, who may not be aware that they have been infected and thus will not seek any medical intervention. Also, there are cases that may have been diagnosed false negative during the screening procedures [8]. Serological (antibody) tests that include immunofluorescence assays, enzyme-linked immunosorbent assays and western blot analysis have emerged as an alternative or complement to RT-PCR. A clear advantage of these tests over RT-PCR is that they can identify individuals previously infected by SARS-CoV-2, even if they never underwent testing while being acutely ill [7].

Majority of the cases that reported in past few months have a history of COVID-19 infection, and in cases where the patient's history is misleading or not suggestive of COVID-19 infection, serological investigations can help clear the dilemma. Currently, two types of antibody testing are being done mainly: binding antibody detection and 
neutralising antibody detection tests. The results can be interpreted as: testing positive for antibody against $\mathrm{N}$ (nucleocapsid protein), S (spike protein) or RBD (receptor binding domain) in a non-vaccinated person indicates a prior infection. In a vaccinated person, testing positive for antibody against $\mathrm{N}$ (nucleocapsid protein) indicates a prior infection as the vaccine produces antibodies only against spike protein. Although these serological tests are not approved by FDA, but these have received EUA (Emergency Use Authorisation) for public health and clinical purposes [9].

Only a few incidental case reports and studies on COVID-associated mucormycosis have been reported. Most of this literature has succeeded in establishing an association of mucormycosis with COVID-19 and DM. However, there are no reliable data to analyse its association with the severity of COVID-19 infection. Also, the evidence in undiagnosed COVID-19/ asymptomatic carriers and non-diabetic patients is lacking. This correlation between severity of COVID-19 infection and mucormycosis may help in better understanding of the disease and formulate prophylactic measures for future control of the disease.

Hanley et al. [10] gave the description of first histopathologically proven case of CAM by post-mortem diagnosis. Following this, various case reports from different parts of the world were published including those by Werthman-Ehrenreich, Mehta et al., Monte Junior ESD et al., Placik et al., Pasero et al., Gard D. et al. and WaizelHaiat S. et al. Almost all of these cases had severe COVID19 infection and the cases succumbed to the disease [11]. An incidence of invasive fungal infections of $26.7 \%$ was reported by White et al. in 135 screened adults with COVID-19 infection. This study concluded that fungal infections frequently occur in severe cases of COVID-19 such as in mechanically ventilated patients [12]. In a recent review article, $8 \%$ of COVID-19 patients (positive as well as recovered) had secondary fungal or bacterial infections during hospital stay, with widespread use of steroids and broad-spectrum antibiotics [13].

Immune dysregulation caused by COVID-19, the similarity in biological behaviour as SARS-CoV, the virus's propensity for ACE-2 receptors in nasal mucosa, overexpression of pro-thrombotic CX3CL1 marker, the virus's tendency to initiate diabetes, the medicines given during COVID-19 or the continued use of same mask are some of the hypotheses proposed in the literature for the pathogenesis of COVID-19-associated mucormycosis (CAM) [14].

In our study, $56 \%$ of the mucormycosis cases had a history of mild COVID-19 infection, $27 \%$ were moderate severity cases and $17 \%$ were severe cases. It is mandatory to stress that all the cases that were categorised as mild were either entirely asymptomatic or were home-isolated. These patients were provided only symptomatic treatment for COVID such as antihistamines, acetaminophen and multivitamins. These patients were never hospitalised during the COVID-19 infection or administered oxygen, steroid or immunomodulators. This finding is in contrast to most of the literature which suggests the occurrence of mucormycosis in severe cases of COVID-19 infection.

Twenty-seven percentage of our patients had a moderate infection of COVID-19 with either SpO2 levels between 90 and $94 \%$ on room air or respiratory rate more than or equal to 24 breaths/ minute. These patients were administered steroids that were probably the reason for hyperglycaemia following the infection. Similarly, $17 \%$ patients had severe COVID-19 infection with either respiratory rate $>30$ breaths/ minute or severe respiratory distress or $\mathrm{SpO} 2<$ $90 \%$ on room air. These patients were tended by oxygen therapy with conservative fluid management and with drugs such as steroids and anticoagulants.

The observations are in contrast to the previous literature. This difference may be attributed to the fact that the present study was done on patients who had recovered from COVID-19 and reported to the OPD services. It is expected that a majority of the severely affected patients may not have survived or must be debilitated or bed-ridden after COVID-19 and may not be in a state to attend OPD services. However, it is interesting to note that a large number of mild cases of COVID-19 acquired mucormycosis, in spite of the treatment sought or hospitalisation.

$5.44 \%(\mathrm{n}=8)$ cases did not test positive for the antibody test. This may be because prior to COVID-19 pandemic also, there was an incidence of mucormycosis cases in our institution. Another justification can be the time period at which the patient reported post-COVID-19 infection, i.e. the patient may have reported after the antibody response had weaned off. Moreover, these serological tests lack $100 \%$ sensitivity and specificity, which could define the results.

Importantly, DM is the most common risk factor associated with mucormycosis globally. So, a reasonable explanation in these cases to acquire CAM may be a rise in the glycaemic levels. India being a country with one of the highest load of diabetes and pre-diabetic population is at risk of secondary infections after the COVID-19 pandemic. $42.9 \%$ of the patients had a pre-existing diabetes at the time of COVID-19 diagnosis, $36.7 \%$ were diagnosed or acquired DM at the time of COVID-19 infection, and $20.4 \%$ were non-diabetic. As quite a large proportion was diagnosed with DM recently, it may be assumed that they were in undiagnosed pre-diabetic stage at the time of COVID infectivity and this condition got stimulated due to the effect of COVID-19 on pancreas. Also, the effect of steroids such as methylprednisolone or dexamethasone 
administered in moderate and severe COVID cases may have precipitated hyperglycaemia and immunosuppression. So, DM remains an independent risk factor for mucormycosis in all the categories of COVID-19 infectivity.

As a significant proportion of CAM are observed in the mild disease category and in non-diabetics, it may be postulated that rather than the factors like steroids, diabetes or ICU stays, it is the COVID-19 virus, that is responsible for mucormycosis. This effect is probably mediated through inflammation and microvascular thrombosis that occurred in COVID-19 [15].

Timely treatment by surgical or endoscopic debridement of the necrotic tissue and appropriate antifungal drugs like Amphotericin B and Posaconazole is essentially helpful in significantly reducing the morbidity and mortality [5]. Optimisation of co-morbidities like DM is another important factor, which needs to be assessed promptly.

The results of the present study confine a high incidence of CAM in mild COVID-19 infected patients also. Although DM is a major risk factor to acquire this fulminant fungal infection, non-diabetics are also at risk.

Medications used and hospitalisation during management of COVID play a negligible role in aggravating invasive fungal infection. So, it is imperative that all the physicians involved in care of COVID-infected patients pay attention to high probability of secondary mucormycosis in post-recovery phase even in those suffering from mild or moderate symptoms. More studies are required to study the causal relationship between COVID-19 and mucormycosis at a molecular level. Also, there is a need to reframe the treatment guidelines during management of mild cases of COVID-19 infection from preventive point of view of invasive fungal disease.

\section{Acknowledgements None}

Funding We did not receive funding from any source for this research

\section{Declaration}

Conflict of interest The authors declare that they have no conflict of interest.

Ethical Approval Ethical approval was obtained from the Institutional ethical committee- Biomedical and Health Research Committee with the study protocol number PGIDS/BHRC/21/45.

Patient Consent This was a record analysing study. Consent of patients who were traceable through contact number was obtained. However, it was not possible to get informed consent from all the patients, so confidentiality of the records was maintained throughout the study.

\section{References}

1. Werthman-Ehrenreich A (2021) Mucormycosis with orbital compartment syndrome in a patient with COVID-19. Am J Emerg Med 42:264-e5

2. Rapidis AD (2009) Orbitomaxillary mucormycosis (zygomycosis) and the surgical approach to treatment: perspectives from a maxillofacial surgeon. Clin Microbiol Infect 15:98-102

3. Mehta S, Pandey A (2020) Rhino-orbital mucormycosis associated with COVID-19. Cureus 12(9)

4. Government of India Ministry of Health and Family welfare. Clinical management protocol for COVID-19 (in adults). https:// www.mohfw.gov.in/pdf/UpdatedDetailedClinicalManagement ProtocolforCOVID19adultsdated24052021.pdf [Accessibility verified June 14, 2021]

5. Singh AK, Singh R, Joshi SR, Misra A (2021) Mucormycosis in COVID-19: a systematic review of cases reported worldwide and in India. Diabet Metab Syndrome: Clin Res Rev

6. Skiada A, Pavleas I, Drogari-Apiranthitou M (2020) Epidemiology and diagnosis of mucormycosis: an update. J Fungi 6(4):265

7. Sidiq Z, Hanif M, KumarDwivedi K, Chopra KK (2020) Benefits and limitations of serological assays in COVID-19 infection. Indian $\mathrm{J}$ Tuberc

8. Han D, Li R, Han Y, Zhang R, Li J (2020) COVID-19: Insight into the asymptomatic SARS-COV-2 infection and transmission. Int J Biol Sci 16(15):2803

9. Centers for Disease Control and Prevention. Interim guidelines for COVID-19 Antibody testing. https://www.cdc.gov/cor onavirus/2019-ncov/lab/resources/antibody-tests-guidelines.html. [Accessibility verified June 14, 2021]

10. Hanley B, Naresh KN, Roufosse C, Nicholson AG, Weir J, Cooke GS, Thursz M, Manousou P, Corbett R, Goldin R, Al-Sarraj S (2020) Histopathological findings and viral tropism in UK patients with severe fatal COVID-19: a post-mortem study. The Lancet Microbe 1(6):e245-53

11. Garg D, Muthu V, Sehgal IS, Ramachandran R, Kaur H, Bhalla A, Puri GD, Chakrabarti A, Agarwal R (2021) Coronavirus disease (Covid-19) associated mucormycosis (CAM): case report and systematic review of literature. Mycopathologia. 1-0.

12. White L, Dhillon R, Cordey A, Hughes H, Faggian F, Soni S, Pandey M, Whitaker H, May A, Morgan M, Wise M (2020) A national strategy to diagnose COVID-19 associated invasive fungal disease in the ICU.

13. Rawson TM, Moore LSP, Zhu N, Ranganathan N, Skolimowska K, Gilchrist M, Satta G, Cooke G, Holmes A (2020) Bacterial and fungal coinfection in individuals with coronavirus: a rapid review to support COVID-19 antimicrobial prescribing. Clin Infect Dis 71(9):2459-2468. https://doi.org/10.1093/cid/ciaa530

14. Agarwal V, Gupta A, Singh V, Jajodia N (2021) Mucormycosis: a post covid-19 enigma. IJCMCR. 10(4): 001. https://doi.org/10. 46998/IJCMCR.2021.10.000241

15. Talasaz AH, Sadeghipour P, Kakavand H, Aghakouchakzadeh M, Kordzadeh-Kermani E, Van Tassell BW, Gheymati A, Ariannejad H, Hosseini SH, Jamalkhani S, Sholzberg M (2021) Recent randomized trials of antithrombotic therapy for patients with COVID-19: JACC state-of-the-art review. J Am Coll Cardiol 77(15):1903-21

Publisher's Note Springer Nature remains neutral with regard to jurisdictional claims in published maps and institutional affiliations. 